\title{
Body-extension versus body-incorporation: Is there a need for a body-model?
}

\author{
Helena De Preester • Manos Tsakiris
}

(C) Springer Science + Business Media B.V. 2009

\begin{abstract}
This paper investigates the role of a pre-existing body-model that is an enabling constraint for the incorporation of objects into the body. This body-model is also a basis for the distinction between body extensions (e.g., in the case of tool-use) and incorporation (e.g., in the case of successful prosthesis use). It is argued that, in the case of incorporation, changes in the sense of body-ownership involve a reorganization of the body-model, whereas extension of the body with tools does not involve changes in the sense of body-ownership.
\end{abstract}

Keywords Embodiment · Body-model · Sense of body-ownership · Incorporation · Prosthesis · Tool

\section{Introduction: embodiment and prostheses}

This article examines on what basis a clear distinction can be made between bodily extensions on the one hand, and the incorporation of non-corporeal items into the body on the other hand. A more proper distinction than one usually finds between these phenomena is helpful for a more clear-cut and coherent interpretation of a number of experimental results. Therefore, in the first place, it will be argued that a number of experimental results concerning tool-use and the 'incorporation' of objects and tools into the body, would benefit from more conceptual clarity. Second,

\footnotetext{
H. De Preester

Faculty of Fine Arts, University College Ghent, J. Kluyskensstraat 6, 9000 Ghent, Belgium

H. De Preester $(\bowtie)$

Department of Philosophy and Moral Sciences, Ghent University, Blandijnberg 2, 9000 Ghent, Belgium

e-mail: helena.depreester@hogent.be
}

\section{Tsakiris}

Department of Psychology, Royal Holloway, University of London, Egham Surrey TW20 0 EX, UK 
it will turn out that the differences between bodily extensions and real bodyincorporation are more profound than generally realized.

Bodily extensions and incorporation are considered here from the viewpoint of embodiment. 'Embodiment', referring to the bodily aspects of human subjectivity, is a major theme in phenomenological philosophy, cognitive science and interdisciplinary research on bodily experience. The distinction between bodily extension and bodyincorporation will be made from a multidisciplinary perspective. Maurice Merleau-Ponty (1945) made a distinction between the objective body and the phenomenal body, i.e. between the body as a material, physiological entity and the body as lived or experienced. Usually, I have my body at my disposal as a sensorimotor unity, which enables me to act in the world. This unity can break down, due to disease or trauma. When the outward, physical body is damaged, both the objective body and the subjective bodily unity (embodiment) may break down in a very dramatic and visible way. Amputation of a limb or part of it, often also involves mending the body with a replacement part or prosthesis. That humans deal with tools, instruments and gear is a common phenomenon in daily life. The confrontation, however, with a lacking bodypart and a corresponding replacement part is far less common, although many people have to try and get along with a prosthesis in their daily life.

The French philosopher Bernard Stiegler, in his major work on the status of technics and prostheses (Stiegler 1998), invites us to think of human prostheses not as accidental and 'extra' possible features of human life, but as something that inherently belongs to human life as such. "The prosthesis is not a mere extension of the human body; it is the constitution of this body qua 'human'." (Stiegler 1998: 152). In Stiegler's context, a prosthesis is considered very broadly, as anything which does not belong to the biological body but co-constitutes the world inhabited by humans. In this sense, prostheses, or technics in general, constitute an essential characteristic of humans, i.e. humans are essentially defined as prosthetic beings. This claim is not new. Martin Heidegger, in his famous analysis of tool-use (Heidegger 1927), already considers tool-use and technics as essential in the characterization of what is means to be a human or a Dasein. Indeed, most people would agree that humans are defined by culture - and the idea that culture is only made possible by artificial prostheses is easily understood. The core idea of culture as prosthetic, is that we often off-load cognitive and physical work onto the environment. That is, we exploit the environment to reduce cognitive and physical workload by designing or preferring environments that can take over physical work from us, or that can hold and even manipulate information for us. Artificial prostheses support the off-loading of physical and cognitive work to the environment by acting as arches between one's body and the environment (Clark 1999). Artificial prostheses include of course tools, but also writing, as an external memory, can be considered a prosthetic extension (cf., e.g., Plato's Phaedrus-dialogue (2003)). Thus, culture is by definition of the order of prosthetic extensions - a human stripped from everything prosthetic-like is a human stripped from culture.

Nevertheless, this is not a statement about facts, claiming, e.g., that any human should rely on prostheses in order to count as a human being. Rather, the statement is about an inherent possibility that characterizes human beings. More precisely, they are characterised as embodied beings whose boundaries are not fixed, but are both plastic and vulnerable. In other words, the use of prostheses is not merely accidental, 
but reveals an inherent possibility of humans as embodied subjects. It is this nonaccidental possibility of the embodied human subject that is at stake here, together with its differentiation into bodily extension and body-incorporation.

The focus is thus on the roots of the above, cultural possibility, namely bodily extensions and prostheses in a literal, physical sense. In other words, the focus is on the relation between the living and lived body and a dead thing attached to the body (for a heideggerian but embodied perspective on this, cf. De Preester 2009). A prosthesis user describes his experience as follows: "Fitting a dead thing to your live body is and always will be an imperfect process. The most critical thing is establishing a good fit. Unfortunately your body will change over time, so a good fit today may not feel as good tomorrow, then it will feel great the next day. The body changes in subtle ways that only those that wear artificial limbs can imagine." (Murray 2004: 966). In the following sections, two possible relations between the living and lived body and something dead attached to it or held by it are discussed.

In the first section, we consider the similarities and differences between bodily extension and bodily incorporation by referring to the examples of tool-use and the attribution of body-parts to one's body. In the second section, we focus on the sense of body-ownership and the putative role of a pre-existing body-model in body plasticity. In the final section, we provide a tentative conclusion by arguing that, in embodiment, there are both experiential and empirical reasons to distinguish between relations of extensions (e.g., in the case of tool-use) and relations of completion (e.g., in the case of prosthesis use). A body-model functions as a constraint on this reorganisation of embodiment, and therefore on what can count as an extension or as a true body-part.

\section{Incorporation versus extension/body-part versus tool?}

In the psychological and neuroscientific literature on embodiment, there often is no clear distinction between the description of successful tool-use and its consequences on the one hand, and the description of incorporation of a prosthesis, in the case of successful prosthesis use, on the other hand. When we look at these two different classes of experiences, prosthesis use and tool-use, it is not easy to maintain an adequate conceptual distinction between a tool that extends the body, and a prosthesis that is incorporated into the body. In fact, this vagueness is already present in Merleau-Ponty's (1945) Phenomenology of Perception, where he explains that the blind man's repeated use of the cane results in the cane becoming "incorporated" (Merleau-Ponty 2002: 139) into the man's body-image (ibid: 141), but also that the cane becomes "a bodily auxiliary, an extension of the bodily synthesis" (ibid: 153, italics added).

In the same line of reasoning, it is usually acknowledged that there is a need to transform the prosthesis from external, added object into bodily part. "For instance, Scarry (1994) reports that the medical community working with prosthesis users often talk of the need to transform the prosthetic limb from an 'inert supplement' or an 'extracorporeal structure' into a corporeal one." (Murray 2004: 964). Instead of remaining a mere tool, the prosthesis should become incorporated, it should become 
a part of the body. This can only happen under certain conditions, and one of them is that the prosthesis becomes a knowing body-part, in other words, something that shares in the knowledge of the body. However, this possibility remains very limited in the case of prostheses. This is shown by the following experience of a one-handed piano student who forgot to take her left prosthetic hand with her when she went for a piano lesson. Her teacher asked her to play with her right hand, and to imagine what the left hand would be doing. However, RH did not succeed and gave up: "I couldn't think about the left hand, because it's not there. I've never had it and I can't think about the left hand. I've no experience. And there's an old Chinese proverb, 'I do and I understand, I don't do and I don't understand.' " (Murray 2004: 969). Nevertheless, the prosthetic left hand also contributes to her corporeal knowledge when she says: "With the prosthesis, the bit I do understand is holding the hymn book, that [the prosthesis] can hold a hymn book, now I know what it feels like to hold a hymn book in the left hand." (ibid.).

The possibility that the prosthesis becomes a knowing body-part is not excluded, although it remains limited. Moreover, it is not sufficient for something to withdraw into the sensorium of the body (cf. Merleau-Ponty's example of the blind man's stick) or to share into the bodily knowledge of the body (which is often motor based or body schematic, cf. the example above) in order to become incorporated. The reason is that tool-use shares these characteristics of withdrawal into the sensorium of the body (cf. Maravita and Iriki 2004; Maravita et al. 2002) and of sharing into the knowledge of the body, but tools do not become part of one's body in the sense of incorporation.

The following quote from a male with congenital absence of the foot, precisely describes the experience of being percipient at the boundaries of the prosthesis, but he also mentions something more, viz. the absence of the prosthesis from his field of attention. "One of the major factors in my satisfaction with a new prosthesis is how little I feel it. That may sound strange, but to me, my prosthesis is an extension of my body. (I can actually 'feel' some things that come into contact with it, without having to see them [...]). It must 'feel' as close to not being there as possible." (Murray 2004: 970). The absence of explicit experience of the limb, or rather the experience that the artificial limb has become transparent, is a phenomenon well observed for original body-parts (cf. Merleau-Ponty 1945). However, this gives rise to the question as to whether this additional characteristic is sufficient for a prosthesis to count as 'incorporated'. A tool can also feel close to not being there; for example, during successful tool-use, the tool is 'ready-to-hand', unnoticed too (cf. Heidegger's analysis of tools in his Being and Time).

Heidegger's analysis finds support in recent neuroscientific research on tool-use.

As shown by the work of Iriki et al. (1996), macaque monkeys can be trained to use a rake to retrieve food from far space. At the behavioral level, the very possibility of using effectively the tool suggests that the tool has extended the animal's reaching distance. At the neural level, bimodal neurons, that is neurons responding to both visual and somatosensory stimuli at or near the hand, have specific visual receptive fields that follow the movements of the hand in space. Importantly, after training in tool-use, the receptive fields of these neurons expanded and finally included the entire length of the tool, suggesting that now the neurons represented the space that was accessible with the rake. Analogous findings have 
been reported in humans using different experimental tasks where the main hypothesis suggested that if we can show that reaching behavior with tools mimics the behavior of reaching with hands alone in the reachable space, then that would suggest that "the tool became incorporated into a putative brain representation of the hand wielding it and that visual stimuli presented at the tip of the tool might be coded by the brain in a similar fashion to those presented directly at the hand" (Maravita and Iriki 2004: 82). Most studies on humans report changes in visual or visual-tactile processing or space perception (e.g. Holmes et al. 2007; Farne et al. 2007; Serino et al. 2007; Witt et al. 2005), rather than on body schema per se. A direct way to test whether tool-use affects the body schema would be to record and contrast kinematic parameters of free hand movement before and after tool-use (for this approach see Cardinali et al. 2008). Overall, what the experiments on tool-use by human and non-human primates claim to show is that alterations in motor capabilities (e.g. reaching into far space with tools) are accompanied by specific changes in the receptive fields of bimodal neurons that may maintain an on-line map of body location and body posture, what in classical neurology has been termed body-schema. These neural changes probably are responsible for the experience of 'withdrawal' of the tool. Tool-use results into a feeling of transparency in which the tool co-enables our experience but is itself not in the focus of our experience. The inconspicuousness of the tool therefore is strongly related to a feeling of nonmediation between the agent and the world.

Berlucchi and Aglioti (1997) call this the extension of the body schema to include non-corporeal objects, or the inclusion of inanimate objects into the body schema: "The body schema can be extended to include non-corporeal objects that bear a systematic relation to the body itself, such as clothes, ornaments and tools. Published examples range from the feather in the hat of Edwardian women to the surgeon's knife and so one. These inclusions of inanimate objects into the body schema are generally regarded as temporary and contingent on the actual association between body and object: when the cyclist dismounts from his bike this ceases to be part of his body schema." (Berlucchi and Aglioti 1997: 561). Others, such as Maravita and colleagues (2003) use another terminology. At first, the emphasis is on the modification of peripersonal space by tool-use. Later on, the following is stated: "This extension of the visual RF [receptive fields] of multisensory neurons following tool-use seems to indicate that previous introspective, or purely speculative, claims that the 'body schema' can extend along a wielded tool or along frequently used objects may in fact have some correspondence to neurobiological reality. [...] Our results, therefore, suggest that with prolonged use, the tool effectively becomes and extension of the hand that wields it [...]." (Maravita et al. 2003: 536, italics added).

The above examples illustrate that the difference between incorporation and extension, and thus between tools and body-parts, and between tools and prostheses, is most often blurred. Nonetheless, Botvinick (2004) pleads indirectly for a distinction between body extensions and body-parts or incorporation: “ [...] during tool use, neurons with body-part-centered visual receptive fields [...] are activated when objects approach not only the hand but the tool itself. From this finding, we would predict that tools are represented as belonging to the bodily self. However, although this may be true in some weak sense, the feeling of ownership that we have 
for our bodies clearly does not extend to, for example, the fork we use at dinner. Apparently, the activation of neurons with (usually) body-part-centered receptive fields may not be entirely sufficient to evoke a feeling of ownership." (Botvinick 2004: 783).

Do the experience and the mechanisms of (body-) ownership offer a way out? The first question is whether (body-) ownership is adequate for making a distinction between incorporation and extension. The second question is whether the distinction can be made solely upon the basis of sensation without taking into account the role of cognitive, that is non-sensorial, body representations.

\section{Body-ownership and a pre-existing body-model}

One way to approach these questions is to consider the recent empirical evidence on the sense of body-ownership, that is, the feeling that a body-part belongs to me. Traditionally, empirical research on body-ownership was hindered by the simple fact that it is very difficult to manipulate body-presence. In classic psychological experiments, the main research approach involves a direct manipulation between conditions where the factor of interest (e.g. my body) is either present or absent. Since my body is "always there, present with me" (James 1890), direct manipulations of body-presence seemed difficult. However, a recent bodily illusion that uses multisensory stimulation to evoke a sense of body-ownership over a fake hand provided a viable way of experimenting with body-ownership. In the Rubber Hand Illusion (Botvinick and Cohen 1998; Ehrsson et al. 2004; Tsakiris and Haggard 2005), participants watch a prosthetic rubber hand being stroked in synchrony with stroking of their own unseen hand. Synchronous, but not asynchronous, multisensory stimulation causes the rubber hand to "feel like it's my hand" (Botvinick and Cohen 1998; Tsakiris and Haggard 2005), to become incorporated in the participant's representation of her own body. This effect suggests that multisensory evidence can be used to produce a subjective feeling of body-ownership for external objects such as the rubber hand. Importantly, once the sense of body-ownership over the rubber hand is evoked, people do not feel as if they have three hands. As shown in a careful analysis of the experience during the RHI (Longo et al. 2008), people tend to feel that the rubber hand is replacing their own hand in the sense that their own hand seems to disappear and be replaced by the rubber hand. It therefore seems that the RHI is not a case of addition or extension of one's body, but instead it is a case of incorporation. A recent study by Moseley et al. (2008) provides direct evidence that the experience of a sense of ownership over a fake hand is accompanied by significant changes in the homeostatic regulation of the real hand. In particular, skin temperature of the real hand decreased when participants experienced the Rubber Hand Illusion. This effect was not evoked by the mere presence of synchronous visual and tactile stimulation, but occurred as a result of the experience of ownership. Thus, ownership over an artificial body-part has direct consequences for real body-parts. These consequences are not simply the result of multisensory integration. Instead, cognitive representations of the body modulate multisensory processing and physiological regulation in a top-down manner. 
Converging evidence (Botvinick and Cohen 1998; Ehrsson et al. 2004; Tsakiris and Haggard 2005) suggests that multisensory stimulation drives the experience of body-ownership. One question that has received considerable interest is whether multisensory stimulation drives body-ownership as a necessary or sufficient condition. If body-ownership was driven by synchronous multisensory stimulation as a sufficient condition, then one would expect to induce a sense of body-ownership over objects that do not resemble body-parts. Interestingly though, replacing the realistic rubber hand with a neutral, non-corporeal, object abolishes the positive effect of synchronous stimulation (Tsakiris and Haggard 2005, see also Graziano et al. 2000, but cf. Armel and Ramachandran 2003). Thus, the effect of multisensory stimulation on body-ownership is not simply a passive stimulus-driven process, but rather seems to depend on the modulatory influence of visual, tactile and postural representations of the body (Graziano and Botvinick 2001; de Vignemont et al. 2006). For example, the RHI is not induced when the rubber hand is placed in an incongruent anatomical posture with respect to one's own hand (i.e. coding of postural representations of body-parts, see Costantini and Haggard 2007; Tsakiris and Haggard 2005, see also Graziano et al. 2000; Pavani et al. 2000; but cf. Armel and Ramachandran 2003), or when the rubber hand is of a different laterality with respect to one's own stimulated hand (i.e. coding of anatomical representations of body-parts, see Tsakiris and Haggard 2005) or when the rubber hand is replaced by a neutral non-corporeal object such a wooden stick (i.e. coding of visual representations of body-parts, see Tsakiris and Haggard 2005; Tsakiris et al. 2008).

On the basis of this evidence, Tsakiris and Haggard (2005) suggested that bodyownership arises as an interaction between multisensory input and body representations. Current sensory stimuli are processed and finally tested-for-fit against an abstract bodymodel that maintains a coherent sense of one's own body. This abstract body-model would contain a reference description of the visual, anatomical and postural properties of one's own body (Costantini and Haggard 2007; Tsakiris and Haggard 2005), that are maintained through time, in contrast to the body-schema model, which is continuously updated as the body moves (Wolpert et al. 1998). Other authors have suggested the existence of a stored and not stimulus-evoked body-structural description that would contain structural representations about (a) the shape and contours of the human body, (b) a detailed plan of the body surface, (c) the location of body-parts, the boundaries between them, and their internal part-relation. Schwoebel and Coslett (2005) suggested that this body-structural description is view-independent or even allocentric, but what seems to be important for the sense of body-ownership induced during the RHI is that the body-model operates off-line and more interestingly it seems to be normative for one's own body, because its modulatory influence allows for an external body-part to be experienced as part of my body or not.

Recent studies suggest that the right temporal and parietal lobes may underpin this body-model. Lesions in this region may result in denial of ownership of the contralateral hand (Bottini et al. 2002), neglect of the left side of the body (Mort et al. 2003) and anosognosia for hemiplegia (Berlucchi and Aglioti 1997). Direct electrical stimulation of the right temporo-parietal junction (rTPJ) in a neurosurgical patient elicited experiences of seeing her body from an external perspective ('out-ofbody experience'), and of illusory transformations of the arms and legs (Blanke et al. 2002). Based on these results, rTPJ may be the neural source of this model of one's 
body against which multisensory stimuli are tested. To test this hypothesis, Tsakiris et al. (2008), delivered transcranial magnetic stimulation (TMS) immediately after synchronous visuo-tactile stimulation to investigate the role of this area in the processing of sensory events during RHI (Tsakiris et al. 2008). Overall, TMS over rTPJ reduced the extent to which the rubber hand was incorporated into the mental representation of one's own body, and it also increased the incorporation of a neutral object (e.g. a spoon), as measured by the proprioceptive drift towards or away from the viewed object. An object (i.e. a rubber hand) that would normally have been perceived as part of the subject's own body was no longer significantly distinguished from a clearly neutral object, suggesting that the disruption of neural activity over rTPJ blocked the contribution of the body-model in the assimilation of current sensory input, making the discrimination between what may or may not be part of one's body ambiguous.

In a follow-up analysis of the results presented in Tsakiris et al. (2008), it is shown that the effects of disrupting rTPJ was strongest when modulation by the body-model is strongest. The contribution of the body-model to the RHI was estimated as the difference between the proprioceptive drifts when viewing a rubber hand and when viewing a neutral object in the control trials. The effect of TMS over rTPJ was estimated as the difference between proprioceptive drifts when TMS was delivered over rTPJ while viewing the rubber hand, and trials without TMS while viewing the rubber hand in the same block. A linear regression analysis (see Fig. 1) showed a significant relation between the two measures $(t(8)=4.15, p<0.05)$.

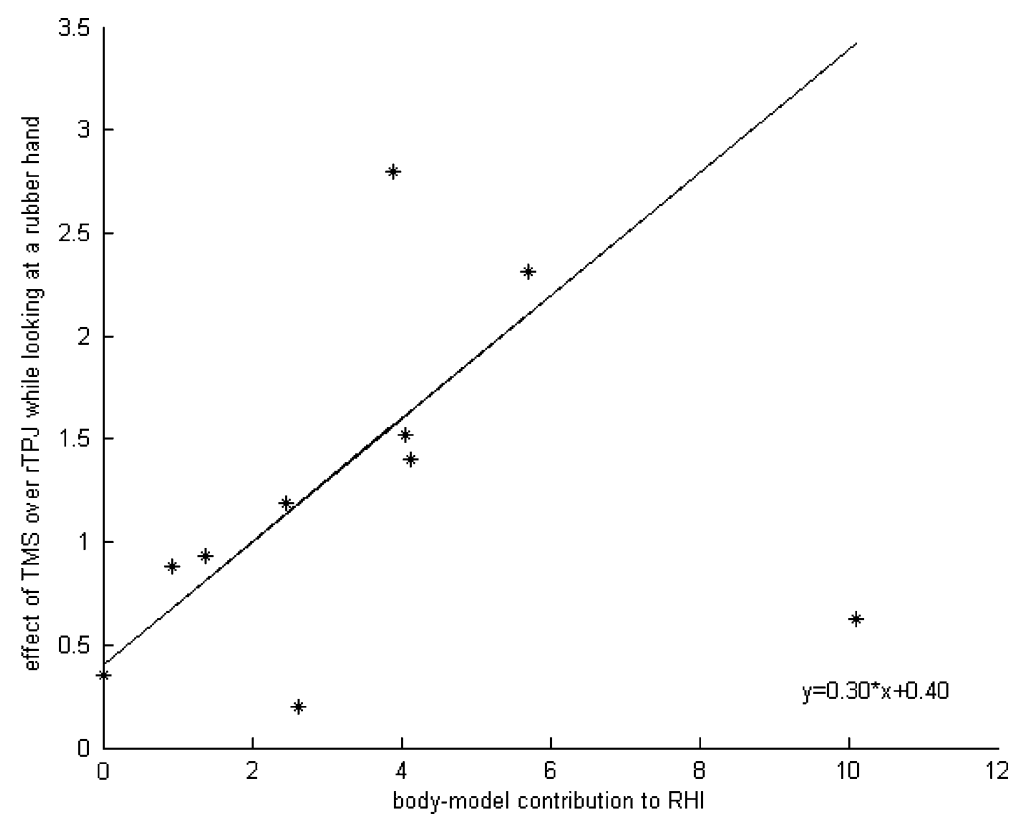

Fig. 1 Correlation across subjects between the effects of TMS over rTPJ while viewing a rubber hand and the body-model contribution to RHI. The line shows a robust weighted regression fitted to the data. From Tsakiris et al. (2008) 
Participants who showed strong discrimination between the effect of viewing the rubber hand vs. a neutral object also showed greater reductions in RHI following TMS over rTPJ, suggesting that the modulation of RHI by TMS over rTPJ was strongly related to the contribution of a body-model to the sense of ownership. This suggests that the contribution of rTPJ in maintaining the sense of ownership over the rubber hand depends on the extent to which subjects use an internal body-model to process current multisensory stimulation.

This finding seems in line with recent proposals regarding one possible function of rTPJ. Decety and Lamm (2007, see also Shulman et al. 2007; for a different view see Saxe and Wexler 2005) suggest that this area may underpin a single computational mechanism that is used by multiple cognitive processes. This basic mechanism involves filtering sensory events for their importance or attribution to the self. The results by Tsakiris et al. (2008) show how the right temporo-parietal junction may underpin this test-for-fit process and maintain a coherent sense of one's body on the basis of multisensory information. This body-model must contain substantial prior information about the body, and the fit between current stimulation and this model can therefore provide a criterion for distinguishing between ownership and disownership.

In summary, the limits of bodily plasticity seem to point to the stability of a preexisting, probably normative, model of the body. The possibility of bodily extension is limited since it most probably is constrained by the top-down influence of this preexisting body-model. This normative model for one's own body does not seem to be sense-specific nor modality-bound (cf. also its use for multiple processes), and functions as a constraint on bodily extension.

Let us have a closer look at the possible existence of a pre-existing body-model, responsible for a certain permanent matrix for the body, in contrast to the more dynamic qualities of the moving and acting body. Carruthers (2008) recently presented an account of two different types of body representations that differ in terms of their origins and content. Online representations are generated directly by current sensory input and represent "what the body is currently like", allowing thus an online control and monitoring of body-configurations. Offline representations are constructed by online representations and represent "what the body is usually like". Offline representations, therefore, code for more permanent and diachronic aspects of the body, such as its structure and possibly its usual appearance and motor repertoire. On this account, online representations are constructed directly from current sensory input and some of these online representations are eventually taken offline. If, as Carruthers suggests, offline representations are not pre-existing, but are serially constructed by online representations, then we can only think of offline representations as the reservoir of accumulated representations of bodily experiences to which we have been repeatedly exposed during ontogenetic development. Accordingly, it is not possible to form an offline body representation unless we had a prior experience that allowed the formation of an online representation of the body-part or sensation that is eventually represented offline. If that was the case, then we would not be able to account for the experience of phantom limbs in patients who congenitally lack a limb or part of it, or for early demonstrations of hand-mouth coordination in utero and early infancy (Gallagher et al. 1998) or, arguably, for the infant's sensitivity to left-right reversal of their own legs shown on a screen (Rochat 
and Morgan 1995). In relation to the phantom sensations experienced by phocomelic children, Berlucchi and Aglioti (1997: 564) write : “(...) phantom sensations can point to the possible existence of a kind of prototypical human body, an ideal model, that has precedence over the real state of the body. Phantom sensations reported by phocomelic children who were born without one or more limbs, as well as the visuomotor imitation abilities of newborn babies, similarly suggest that the brain might be genetically predisposed to represent a prototypical human body, regardless of the correspondence or lack thereof between ideal model and actual body."

An elegant experiment on normal infants by Morgan and Rochat (1997) support the idea of an innate body-model. Morgan and Rochat presented 3-, and 4- to 5-month-old infants with an online (ego) view of their own legs, which kept both orientation and movement directionality of either legs constant, but altered their relative position on the screen. In the normal condition, infants saw their legs in their normal relative positions: the right leg to the right of the screen and the left leg to the left. In the reversed condition, the legs positions were reversed: the left leg to the right and the right leg to the left side of the screen. The results showed that from at least 3 months of age, infants manifest differential looking and kicking behavior across conditions. Infants reduced their looking and leg activity when presented with a reversed relative location of their legs on the screen, suggesting that young infants, with relatively small experience of seeing their legs, are sensitive to differences in the relative movements and/or the featural characteristics of the legs (i.e., the relative bending of the legs at the knees and ankles), supporting thus the idea of an innate body-model that represents anatomical and structural features of a normative body.

The evidence reviewed above suggests that at least some body representations seem to be hardwired and innate, challenging thus the idea that offline representations derive only from online representations. The idea of innate representations suggests that at least some anatomical features of the body (e.g. number of limbs, left vs. right limbs) are a priori represented, but the actual features of body-parts (e.g. morphology, length of a limb) may be constructed and updated throughout development.

\section{Tentative conclusions}

The existence of a body-model that normatively constrains what can count as a body-part gives us the basis for a distinction between body-incorporation and bodyextension. Incorporation of non-bodily objects into body representations would involve changes in motor and perceptual capacities, but also in the feeling of bodyownership. Moreover, these non-corporeal objects have to fit with a pre-existing, normative model of the body that regulates which objects sufficiently resemble body-parts, based on postural, anatomical and visual clues.

The question of whether incorporation of non-corporeal objects also implies an extended sense of body-ownership probably has to be answered negatively. Changes in the sense of body-ownership rather point into the direction of an internal reorganisation of body-ownership, rather than an extension of it. A feeling of bodyownership is only attributed to body-parts that can be assimilated in a pre-existing body-model, which is as such normative for the feeling of ownership. This means 
that something originally non-corporeal can only belong to the body-model if it replaces something that already belonged to the body-model. Therefore, changes in the feeling of body-ownership probably can only happen on the basis of replacing older body-parts with items that will count as body-part when they are taken up into the body-model.

The body-model or pre-existing frame of reference for one's own body is in this sense also responsible for a kind of body constancy. The reorganization of the bodymodel and of the feeling of body-ownership always is a matter of internal reorganization: parts can be replaced with non-corporeal items, but the body-model as a whole cannot be extended; it an only be internally reorganized.

In contrast, extensions of body representations with non-corporeal objects, i.e. tools, involve (bottom-up) changes in motor and perceptual capacities, but without changes in body-ownership. In the case of bodily extensions, the phenomenology of the bodily changes would not be a relation of replacement, but a relation of addition. This relation between body and tool has more degrees of freedom than the relation of replacement, i.e. the re-organisation of body-ownership, which is constrained by the body-model. This means that the feeling of embodiment can be extended with tools, and that the body-model is not a normative constraint for extending the body with tools. As such, the relation between body and tool remains more external than the uptake of a new body-part by means of changes in body-ownership and internal reorganisation of the body-model.

An important difference between extension of the body with a tool and replacement of a body-part with something non-corporeal, is the possible experience of completion. Ideally, the relation between a human and his or her prosthesis is experienced as a relation of completion. In other words, it is possible to make a whole with the prosthesis that substitutes a missing limb or part of it. That prostheses can complete a body, i.e. that they can become 'part of' the body, is testified by both amputees and people with congenital limb absence. "Well, to me it's as if, though I've not got my lower arm, it's as though I've got it and it's (the prosthesis) part of me now. It's as though I've got two hands, two arms." (Murray 2004: 970). We do not make a whole with tools in the same way. A tool does not feel as if it is completing us, but it rather extends bodily capacities.

BIID (body integrity identity disorder), in which a subject has "a feeling of 'incompleteness and disability' as a four limbed individual but a certainty of a feeling of 'completeness and enablement' after amputation" (cf. www.biid.org, the official website for BIID) might be related to a dysfunctional body-model, in which the norm for the feeling of completeness is defined along other dimensions or in which the body-model is organized differently (e.g. not two lower limbs down from the knee, but only one).

However, this does not mean that all prosthesis users do or should experience a relation of completion with their prosthesis. Often, they experience a relation of extension, as reported by the following subject. "Using a prosthetic is not a natural thing, because a prosthetic is not a substitute leg, it is a tool which may or may not do some of the things that a leg might have done." (Murray 2004: 971).

On the other hand, the question if tools can become part of the body, or if a relation of extension can be turned into a relation of completion, cannot be definitely settled by answering negatively. Although it seems difficult and at this moment 
rather improbable to make the body-model accept a 'mere' extension as a body-part, since there is no internal reorganisation of the body-model involved, some very specialized tool-users, such as musicians, report about the relation between themselves and their instrument as an experience of completion or wholeness. One of the reasons may be an aspect of tool-use not touched upon in this article, namely the fact that some tools, such as music instruments, allow (and are designed for) expression. The experience of wholeness with a music instrument might partly be due to this possibility of highly extending the expressive body (and not just the body as a sensorimotor body). The question what exactly can induce a relation of completion is of course not irrelevant in a highly technological and 'cybernized' society. Whatever the further evolution between humans and their extensions or prostheses, it is highly probable that a normative body-model that shapes these relations will have to be taken into account.

Acknowledgments HDP was supported by a research grant by the University College Ghent. MT was supported by the "European Platform for Life Sciences, Mind Sciences, and the Humanities" grant by the Volkswagen Stiftung for the "Body-Project: interdisciplinary investigations on bodily experiences".

\section{References}

Armel, K. C., \& Ramachandran, V. S. (2003). Projecting sensations to external objects: Evidence from skin conductance response. Proceedings of the Royal Society of London: Biological, 270, 1499-1506.

Berlucchi, G., \& Aglioti, S. (1997). The body in the brain: Neural bases of corporeal awareness. Trends in Neurosciences, 20, 560-564.

Blanke, O., Ortigue, S., Landis, T., \& Seeck, M. (2002) Stimulating illusory own-body perceptions. Nature, 419, 269-270.

Bottini, G., Bisiach, E., Sterzi, R., \& Vallarc, G. (2002). Feeling touches in someone else's hand. Neuroreport, 13, 249-252.

Botvinick, M. (2004). Probing the neural basis of body ownership. Science, 305, 782-783.

Botvinick, M., \& Cohen, J. (1998). Rubber hands 'feel' touch that eyes see. Nature, 391, 756.

Cardinali, L., Frassinetti, F., Brozzoli, C., Roy, A. C., Urquizar, C., \& Farnè, A. (2008). Functionality dependent tool embodiment. Poster presented at "Sense of Body Summer School", Bologna, June 2008 (retrieved from http://www.senseofbody.eu/Abstract-Sense\%20of\%20Body\%202008.pdf on 15.01.2009).

Carruthers, G. (2008). Types of body representation and the sense of embodiment. Consciousness \& Cognition, 17(4), 1302-1316.

Clark, A. (1999). An embodied cognitive science. Trends in Cognitive Sciences, 3(9), 345-351.

Costantini, M., \& Haggard, P. (2007). The rubber hand illusion: Sensitivity and reference frame for body ownership. Consciousness \& Cognition, 16(2), 229-240.

De Preester (2009) The bodily origins of technics: Heidegger, cognitive science and the prosthetic subject. In J. Kiverstein, \& M. Wheeler (Eds.), Heidegger and cognitive science. Palgrave Macmillan, Houndsmill (in press).

de Vignemont, F., Tsakiris, M., \& Haggard, P. (2006). Body mereology. In G. Knoblich, I. Thornton, M. Grosjean, \& M. Shiffrar (Eds.), Perception of the human body (pp. 147-170). New York, NY: Oxford University Press.

Decety, J., \& Lamm, C. (2007). The role of the right temporoparietal junction in social interaction: How low-level computational processes contribute to metacognition. The Neuroscientist: A Review Journal Bringing Neurobiology, Neurology and Psychiatry, 13, 580-593.

Ehrsson, H. H., Spence, C., \& Passingham, R. E. (2004). That's my hand! Activity in premotor cortex reflects feeling of ownership of a limb. Science, 305, 875-877.

Gallagher, S., Butterworth, G., Lew, D., \& Cole, J. (1998). Hand-mouth coordination, congenital absence of limb, and evidence for innate body schemas. Brain and Cognition, 38, 53-65. 
Farnè, A., Serino, A., \& Làdavas, E. (2007). Dynamic size-change of peri-hand space following tool-use: determinants and spatial characteristics revealed through cross-modal extinction. Cortex; A Journal Devoted to the Study of the Nervous System and Behavior, 43(3), 436-443.

Graziano, M. S. A., \& Botvinick, M. M. (2001). How the brain represents the body : insights from neurophysiology and psychology. In W. Prinz, \& B. Hommel (Eds.), Common mechanisms in perception and action, attention and performance XIX (pp. 136-158). Oxford, NY: Oxford University Press.

Graziano, M. S. A., Cooke, D. F., \& Taylor, C. S. R. (2000). Coding the location of the arm by sight. Science, 290, 1782-1786.

Heidegger, M. (1962 [1927]). Being and time (transl. Macquarrie J, Robinson E). London: : Blackwell.

Holmes, N. P., Calvert, G. A., \& Spence, C. (2007). Tool use changes multisensory interactions in seconds: Evidence from the crossmodal congruency task. Experimental Brain Research. Experimentelle Hirnforschung. Experimentation Cerebrale, 183(4), 465-476.

Iriki, A., Tanaka, M., \& Iwamura, Y. (1996). Coding of modified body schema during tool use by macaque postcentral neurones. Neuroreport, 7, 2325-2330.

James, W. (1890). The principles of psychology. Cambridge: Harvard University Press.

Longo, M. R., Schüür, F., Kammers, M. P. M., Tsakiris, M., \& Haggard, P. (2008). What is embodiment? A psychometric approach. Cognition, 107(3), 978-998.

Maravita, A., \& Iriki, A. (2004). Tools for the body (schema). Trends in Cognitive Sciences, 8(2), 79-86.

Maravita, A., Spence, C., Kennett, S., \& Driver, J. (2002). Tool-use changes multimodal spatial interactions between vision and touch in normal humans. Cognition, 83, B25-B34.

Maravita, A., Spence, C., \& Driver, J. (2003). Multisensory integration and the body schema: close to hand and within reach. Current Biology, 13, R531-R539.

Merleau-Ponty, M. (1945). Phenomenology of perception (transl. C. Smith). London: Routledge.

Morgan, R., \& Rochat, P. (1997). Intermodal calibration of the body in early infancy. Ecological Psychology, 9, 1-24.

Mort, D. J., Malhotra, P., Mannan, S. K., Rorden, C., Pambakian, A., Kennard, C., et al. (2003). The anatomy of visual neglect. Brain, 126, 1986-1997.

Moseley, G. L., Olthof, N., Venema, A., Don, S., Wijers, M., Gallace, A., et al. (2008). Psychologically induced cooling of a specific body part caused by the illusory ownership of an artificial counterpart. Proceedings of the National Academy of Sciences of the USA, 105(35), 13169-13173.

Murray, C. D. (2004). An interpretative phenomenological analysis of the embodiment of artificial limbs. Disability and Rehabilitation, 26(16), 963-973.

Pavani, F., Spence, C., \& Driver, J. (2000). Visual capture of touch: Out-of-the-body experiences with rubber gloves. Psychological Science: A Journal of the American Psychological Society/APS, 11, 353-359.

Plato (2003). Phaedrus (transl. Scully, S.). Newburyport: Focus.

Rochat, P., \& Morgan, R. (1995). Spatial determinants in the perception of self-produced leg movements by 3- to 5-month-old infants. Developmental Psychology, 31, 626-636.

Saxe, R., \& Wexler, A. (2005). Making sense of another mind: the role of the right temporo-parietal junction. Neuropsychologia, 43(10), 1391-1399.

Scarry, E. (1994). The merging of bodies in artifacts in the social contract. In G. Bender, \& T. Druckery (Eds.), Culture on the brink: ideologies of technology (pp. 85-97). Seattle: Bay.

Schwoebel, J., \& Coslett, H. B. (2005). Evidence for multiple, distinct representations of the human body. Journal of Cognitive Neuroscience, 17(4), 543-553.

Serino, A., Bassolino, M., Farnè, A., \& Làdavas, E. (2007). Extended multisensory space in blind cane users. Psychological Science: A Journal of the American Psychological Society / APS, 18(7), 642-648.

Shulman, G. L., Astafiev, S. V., McAvoy, M. P., d'Avossa, G., \& Corbetta, M. (2007). Right TPJ deactivation during visual search: Functional significance and support for a filter hypothesis. Cerebral Cortex, 17, 2625-2633.

Stiegler, B. (1998). Technics and time, 1. The fault of epimetheus. Stanford: Stanford University Press.

Tsakiris, M., \& Haggard, P. (2005). The rubber hand illusion revisited: visuotactile integration and selfattribution. Journal of Experimental Psychology: Human Perception and Performance, 31, 80-91.

Tsakiris, M., Costantini, M., \& Haggard, P. (2008). The role of the right temporo-parietal junction in maintaining a coherent sense of one's body. Neuropsychologia, 46(12), 3014-3018.

Witt, J. K., Proffitt, D. R., \& Epstein, W. (2005). Tool use affects perceived distance, but only when you intend to use it. Journal of Experimental Psychology. Human Perception and Performance, 31(5), 880-888.

Wolpert, D. M., Goodbody, S. J., \& Husain, M. (1998). Maintaining internal representations: the role of the human superior parietal lobe. Nature Neuroscience, 1(6), 529-533. 